\title{
BCAT1 overexpression regulates proliferation and c-Myc/GLUT1 signaling in head and neck squamous cell carcinoma
}

\author{
HONGMING WANG, FEI WANG, WENYU OUYANG, XUEJUN JIANG and YAN WANG \\ Department of Otolaryngology, The First Affiliated Hospital of China Medical University, \\ Shenyang, Liaoning 110001, P.R. China
}

Received May 28, 2020; Accepted November 27, 2020

DOI: $10.3892 /$ or.2021.8003

\begin{abstract}
Branched chain amino acid transaminase 1 (BCAT1) overexpression has been reported in various cancers; however, at present, its significance and biological role in head and neck squamous cell carcinoma (HNSCC) remain unknown. BCAT1 protein expression was upregulated in 56/106 (52.8\%) cases of HNSCC. BCAT1 overexpression was associated with tumor-node-metastasis stage, tumor stage and nodal metastasis. The Cancer Genome Atlas data suggested that high BCAT1 expression was associated with poor patient survival. Oncomine data suggested that BCAT1 expression was increased in HNSCC. Functionally, BCAT1 overexpression promoted cell proliferation, colony formation, invasion and cisplatin resistance in FaDu cells. BCAT1 overexpression also upregulated the mitochondrial membrane potential, and increased ATP production, glucose consumption and glucose uptake. Western blotting demonstrated that BCAT1 overexpression upregulated c-Myc and glucose transporter 1 (GLUT1) protein levels. Depletion of c-Myc using small interfering RNA abolished the influence of BCAT1 on GLUT1. Chromatin immunoprecipitation assays demonstrated that c-Myc has binding sites in the GLUT1 promoter. Collectively, the present findings suggested that BCAT1 is upregulated in human HNSCC and regulates HNSCC cell proliferation, invasion, cisplatin sensitivity and c-Myc/GLUT1 signaling.
\end{abstract}

\section{Introduction}

Head and neck squamous cell carcinoma (HNSCC) is the sixth-most common malignancy worldwide, with 890,000 new cases and 450,000 deaths in $2018(1,2)$. Despite progress in treatment strategies, patient survival remains poor, with a 5 -year survival rate of $<50 \%$ (3). Recurrence and metastasis

Correspondence to: Dr Yan Wang, Department of Otolaryngology, The First Affiliated Hospital of China Medical University, 155 North Nanjing Street, Shenyang, Liaoning 110001, P.R. China

E-mail: wangyancmu@126.com

Key words: c-Myc, glucose transporter 1, branched chain amino acid transaminase 1 , head and neck squamous cell carcinoma are frequent events leading to HNSCC mortality (4). Therefore, it is important to identify novel biomarkers and understand the molecular mechanisms of HNSCC progression.

Branched-chain amino acid (BCAA) transaminase 1 (BCAT1) is a BCAA aminotransferase enzyme that catalyzes transamination of glutamate and BCAAs $(5,6)$. Cytosolic BCAT1 expression is restricted to a limited number of tissues, including the brain, ovaries and testes (7). The involvement of BCAT1 in tumor development has been implicated in glioblastoma (8), breast cancer (9) and leukemia $(10,11)$. BCAT1 promotes the proliferation of cancer cells by regulating energy metabolism (10). BCAT1 can promote epithelial-mesenchymal transition in liver cancer (12). BCAT1 promotes the proliferation of breast cancer cells by enhancing mTOR-mediated mitochondrial biogenesis and function (9). These reports thus suggested that BCAT1 is an onco-protein in human cancers. At present, however, its expression pattern and biological characteristics in human HNSCC remain unexplored.

In the present study, BCAT1 expression was examined in HNSCC tissues via immunohistochemistry, and its relationship with clinicopathological factors was analyzed. The effects of BCAT1 on cell proliferation, invasion, chemosensitivity, mitochondrial function and glucose metabolism were evaluated, and the underlying mechanism was explored via chromatin immunoprecipitation (ChIP). The present findings provided novel evidence implicating BCAT1 as an oncogene in HNSCC progression.

\section{Materials and methods}

Samples. The present study was approved by the Institutional Review Board and Ethics Committee of the First Affiliated Hospital of China Medical University (approval no. AF-SOP-07-1.0-01). A total of 106 cases of HNSCC and 23 cases of normal head and neck tissues were obtained from the Pathology Archives of the First Affiliated Hospital of China Medical University (Shenyang, China), which were collected between January 2012 and December 2016. HNSCC samples included 83 male and 23 female patients. The age of the patients ranged between 36-79 years, with an average age of 57.6 years. The normal control group enrolled 10 male and 13 female patients, with an average age of 58.5 years (range, 41-77 years). The patients agreed to the use of their samples in 
scientific research and informed consent was obtained from the participants.

Immunohistochemistry. Paraffin sections ( $4 \mu \mathrm{m})$ were deparaffinized using xylene and a graded alcohol series. $\mathrm{H}_{2} \mathrm{O}_{2}(3 \%)$ was applied to the slides to block peroxidase activity at $37^{\circ} \mathrm{C}$ for $15 \mathrm{~min}$, and the sections were incubated with normal goat serum (Fuzhou Maixin Biotech Co., Ltd.) at $37^{\circ} \mathrm{C}$ for $30 \mathrm{~min}$ to reduce nonspecific binding. Then, the sections were incubated with BCAT1 antibody (1:500; cat. no. 28622-1-AP; ProteinTech Group, Inc.) overnight at $4^{\circ} \mathrm{C}$. Immunohistochemical staining was performed using an Elivision Plus kit (Fuzhou Maixin Biotech Co., Ltd.). Briefly, the sections were incubated with polymer enhancer at $37^{\circ} \mathrm{C}$ for $20 \mathrm{~min}$ and then with horseradish peroxidase (HRP)-conjugated anti-mouse/rabbit IgG. Staining was developed with a DAB kit (Fuzhou Maixin Biotech Co., Ltd.) at room temperature for $3 \mathrm{~min}$. Hematoxylin was used for counterstaining at room temperature for $5 \mathrm{~min}$.

Immunostaining of BCAT1 was evaluated using a semiquantitative scale by assessing both the intensity of staining and the percentage of cells stained. Five fields were examined per slide under a BX53 light microscope (magnification, x400; Olympus Corporation). BCAT1 intensity was scored as 0 (negative), 1 (weak) or 2 (strong). The percentage of cells stained was scored as 1 (1-25\%), 2 (26-50\%), $3(51-75 \%)$ or $4(76-100 \%)$. The intensity and percentage scores were multiplied to obtain a final score of 0-8. Samples were considered to have low BCAT1 expression with final scores $<4$. Samples with scores $\geq 4$ were considered to have high BCAT1 expression.

Bioinformatics analysis. BCAT1 gene expression profiles for patients with HNSCC were obtained from The Cancer Genome Atlas (TCGA; https://tcga-data.nci.nih.gov/tcga/). Clinical data including survival and clinical $\mathrm{T}$ stage were also downloaded from TCGA (13). The expression of BCAT1 in various types of HNSCC was identified in the Oncomine database (https://www.oncomine.org/resource/login.html) (14). Data were analyzed using GraphPad Prism 7.00 software (GraphPad Software, Inc.).

Cell culture and transfection. The FaDu cell line was obtained from the Shanghai Cell Bank of the Chinese Academy of Sciences. Cells were cultured with RPMI-1640 medium (Gibco; Thermo Fisher Scientific, Inc.) with 10\% FBS (Gibco; Thermo Fisher Scientific, Inc.) under $5 \% \mathrm{CO}_{2}$ at $37^{\circ} \mathrm{C}$. pCMV6-BCAT1 plasmid $(1 \mu \mathrm{g})$ and control pCMV6 plasmid (OriGene Technologies, Inc.) were transfected into $\mathrm{FaDu}$ cells (50\% confluence) using Lipofectamine ${ }^{\circledR} 3000$ reagent (Thermo Fisher Scientific, Inc.) for 72 h. BCAT1 small interfering (si)RNA (target sequence, 5'-GUACAAAGGCGA GACAAUA-3') and non-targeting siRNA (target sequence, 5'-UAGCGACUAAACACAUCAA-3'; both GE Healthcare Dharmacon, Inc.) were transfected using DharmaFECT1 reagent (GE Healthcare Dharmacon, Inc.). The final siRNA concentration used for transfection was $0.025 \mu \mathrm{M}$.

Reverse transcription-quantitative $(R T-q) P C R$. TRIzol reagent (Invitrogen; Thermo Fisher Scientific, Inc.) was used to extract RNA from cells. RT was performed with an
PrimeScript RT Master Mix kit (Takara Bio, Inc.) at $85^{\circ} \mathrm{C}$ for $2 \mathrm{~min}$ and $37^{\circ} \mathrm{C}$ for $30 \mathrm{~min}$. qPCR was performed using SYBR Green Master Mix (Thermo Fisher Scientific, Inc.) on a 7500 Real-Time PCR System (Thermo Fisher Scientific, Inc.) at $50^{\circ} \mathrm{C}$ for $2 \mathrm{~min}, 95^{\circ} \mathrm{C}$ for $2 \mathrm{~min}$, and 40 cycles of $95^{\circ} \mathrm{C}$ for $15 \mathrm{sec}$ and $60^{\circ} \mathrm{C}$ for $40 \mathrm{sec}$. qPCR was quantified using the $2^{-\Delta \Delta \mathrm{Cq}}$ method (15) and GAPDH was used as a reference control. The following primers were used: BCAT1 forward, 5'-TGCTAGTCTGTATATTCGTCCT-3' and reverse, 5'-CCA AGAGAAGGCTCAGTTCC-3'; and GAPDH forward, 5'-AAC GACCACTTTGTCAAGCTC-3' and reverse, 5'-AGCCAA ATTCGTTGTCATACCAG-3'.

Cell Counting Kit-8 (CCK-8) and colony formation assays. Cell proliferation was examined using $\mathrm{CCK}-8$ reagent (Dojindo Molecular Technologies, Inc.). Cells were seeded into 96-well plates at 3,000 cells/well and cultured at $37^{\circ} \mathrm{C}$ for $24,48,72$ or $96 \mathrm{~h}$. CCK-8 reagent $(10 \mu \mathrm{l})$ was added to each well. After incubation for $2 \mathrm{~h}$, absorbance was examined at a wavelength of $450 \mathrm{~nm}$ using a microplate reader.

Colony formation assays were performed using 2,000 cells/culture plate. Cells were cultured at $37^{\circ} \mathrm{C}$ for 2 weeks and then stained using Giemsa at room temperature for $10 \mathrm{~min}$.

Western blotting. Total protein was extracted using RIPA lysis and extraction buffer (Thermo Fisher Scientific, Inc.) and quantified via the Bradford method. Proteins (50 $\mu \mathrm{g} / \mathrm{lane})$ were separated via $10 \%$ SDS-PAGE and transferred to PVDF membranes. The membranes were blocked with $5 \%$ milk at room temperature for $1 \mathrm{~h}$ and then incubated with antibodies against BCAT1 (1:1,500; cat. no. 13640-1-AP; ProteinTech Group, Inc.), GLUT1 (1:1,000; cat. no. 12939; Cell Signaling Technology, Inc.), c-Myc (1:1,000; cat. no. 5605; Cell Signaling Technology, Inc.), and GAPDH (1:3,000; cat. no. 5174; Cell Signaling Technology, Inc.). After incubation with HRP-coupled secondary antibodies (1:3,000; cat. no. 7074; Cell Signaling Technology, Inc.), protein bands were visualized using a Pierce ${ }^{\text {TM }}$ Fast Western Blot kit ECL Substrate (Thermo Fisher Scientific, Inc.). Images were captured using a DNR bio-imaging system (DNR Bio-Imaging Systems, Ltd.).

Invasion assay. Transwell invasion assays were performed using Transwell chambers (Corning, Inc.) coated with Matrigel (BD Biosciences) at $37^{\circ} \mathrm{C}$ for $4 \mathrm{~h}$. The treated cells were washed gently with medium without serum, and then placed in the upper chamber at a density of $1 \times 10^{4}$ cells/well. RPMI-1640 medium with $10 \%$ FBS was added to the lower chamber. The Transwell chambers were placed in an incubator at $37^{\circ} \mathrm{C}$ for $24 \mathrm{~h}$. The cells which invaded to the lower wells were fixed in paraformaldehyde at room temperature for $20 \mathrm{~min}$ and then stained with hematoxylin for $5 \mathrm{~min}$ at room temperature. The number of invading cells in random three fields were counted under a BX53 microscope (magnification, x200).

Apoptosis assay and JC-1 staining. FaDu cells were transfected with pCMV6-BCAT1, BCAT1 siRNA or corresponding negative controls. At $48 \mathrm{~h}$ later, the cells were treated with $2 \mu \mathrm{M}$ cisplatin (cat. no. P4394; Sigma-Aldrich; Merck $\mathrm{KGaA}$ ) at $37^{\circ} \mathrm{C}$ for $24 \mathrm{~h}$. Then the cells were stained 
Table I. Association between clinicopathological features and BCAT1 expression in head and neck squamous cell carcinoma.

\begin{tabular}{|c|c|c|c|c|}
\hline Characteristics & Number of patients & Low BCAT1 expression & High BCAT1 expression & P-value \\
\hline Age, years & & & & 0.2677 \\
\hline$<60$ & 59 & 25 & 34 & \\
\hline$\geq 60$ & 47 & 25 & 22 & \\
\hline Sex & & & & 0.3101 \\
\hline Male & 83 & 37 & 46 & \\
\hline Female & 23 & 13 & 10 & \\
\hline Node metastasis & & & & 0.0132 \\
\hline Absent & 77 & 42 & 35 & \\
\hline Present & 29 & 8 & 21 & \\
\hline Tumor stage & & & & 0.0207 \\
\hline $\mathrm{T} 1+\mathrm{T} 2$ & 87 & 46 & 41 & \\
\hline $\mathrm{T} 3+\mathrm{T} 4$ & 19 & 4 & 15 & \\
\hline TNM stage & & & & 0.021 \\
\hline I + II & 64 & 39 & 25 & \\
\hline III + IV & 42 & 11 & 31 & \\
\hline Differentiation & & & & 0.1546 \\
\hline Well & 58 & 31 & 27 & \\
\hline Moderate/poor & 48 & 19 & 29 & \\
\hline
\end{tabular}

BCAT1, branched chain amino acid transaminase 1; TNM, tumor-node-metastasis.

with Annexin V-FITC/PI or JC-1 respectively. The rate of apoptosis was determined using Annexin V-FITC/propidium iodide (PI) staining (BD Biosciences). Cells were incubated with $5 \mu \mathrm{l}$ FITC Annexin V and $5 \mu \mathrm{l}$ PI for $15 \mathrm{~min}$ at room temperature in the dark. The apoptotic rate was calculated as the percentage of early + late apoptotic cells. JC-1 staining was used to determine the mitochondrial membrane potential $(\Delta \psi \mathrm{m})$. The cells were harvested, washed with PBS and incubated with $20 \mu \mathrm{M} \mathrm{JC}-1$ (cat. no. ab113850; Abcam) for $10 \mathrm{~min}$ at room temperature. The cells were analyzed using an ACEA flow cytometer (ACEA Bioscience, Inc.; Agilent Technologies, Inc.). Data were analyzed using NovoExpress version 1.2.4 software (ACEA Bioscience, Inc.; Agilent Technologies, Inc.).

Glucose uptake assay. A 2-NBDG uptake kit (cat. no. K682; BioVision, Inc.) was used to examine the rate of glucose uptake according to the manufacturer's protocols. Glucose uptake were analyzed using flow cytometry at an excitation wavelength of $488 \mathrm{~nm}$. The data were analyzed using NovoExpress.

Glucose consumption assay. Glucose assay kits were purchased from Abcam (cat. no. ab65333). At $72 \mathrm{~h}$ after transfection, the culture medium was collected and centrifuged at $10,000 \mathrm{x} \mathrm{g}$ for $10 \mathrm{~min}$ at $4^{\circ} \mathrm{C}$. The supernatant was collected and glucose levels in the medium were determined using glucose assay kits according to the manufacturer's instructions.

ATP production assay. An ATP assay kit (cat. no. ab83355; Abcam) was used to measure the rate of ATP production. Assays were performed according to the manufacturer's protocols. Briefly, cells were lysed, centrifuged at $13,000 \mathrm{x} \mathrm{g}$ at $4^{\circ} \mathrm{C}$ for $5 \mathrm{~min}$, and filtered using spin columns. Treated samples $(5 \mu \mathrm{l})$ were mixed with ATP reaction mix in 96-well plates. The mixture was measured at excitation/emission wavelengths of 535/587 $\mathrm{nm}$.

ChIP. ChIP assays were performed using a Magna ChIP G Assay kit (EMD Millipore) according to the manufacturer's instructions. Briefly, cells were crosslinked, pelleted and resuspended in lysis buffer, then sonicated. The supernatant was incubated overnight at $4^{\circ} \mathrm{C}$ with c-Myc (1:50; cat. no. 9402; Cell Signaling Technology, Inc.) or IgG antibody (1:50; cat. no. 2729; Cell Signaling Technology, Inc.) and Protein G-magnetic beads. The beads were washed, and the chromatin complexes were collected, purified and de-crosslinked. The DNA fragments were quantified via qPCR analysis. Binding sites for c-Myc in the GLUT1 promoter were predicted using TRANSFAC 7.0 (http://gene-regulation.com/pub/databases. html). The primers for ChIP were as follows: GLUT1 position 1 forward, 5'-GTGGAGAGACTTTGAGGAGGG-3' and reverse, 5'-CACTTGTCCTCTGTCTGCCTT-3'; and GLUT1 position 2 forward, 5'-GTCTCAAGTAAGGCACTGGTC-3' and reverse, 5'-TTCCCACACCAATCTCATTGCT-3'.

Statistical analysis. SPSS 17.0 (SPSS, Inc.) was used for statistical analysis. All experiments were repeated three times. The data were presented as the mean \pm standard deviation. The associations between BCAT1 expression and clinical parameters were analyzed using $\chi^{2}$ test or Fisher's exact test. Kaplan-Meier analysis with log-rank test was used to estimate the overall survival (OS) of patients with HNSCC. 
A

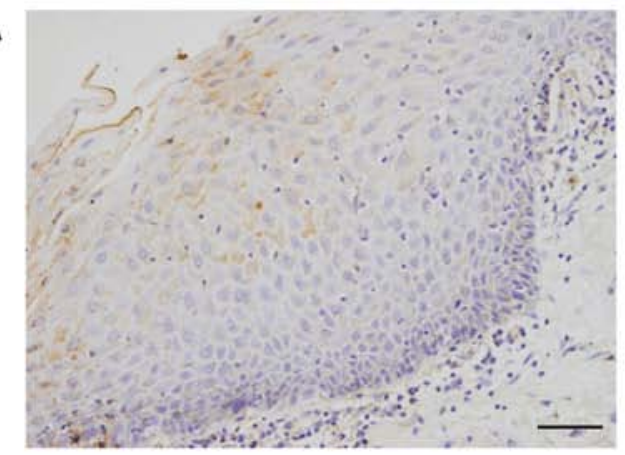

C

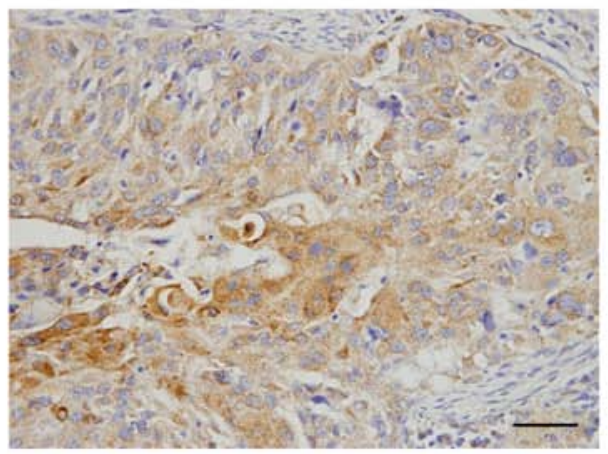

B

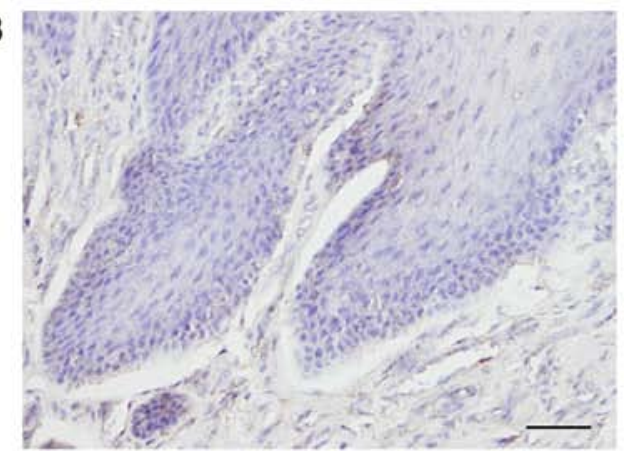

D

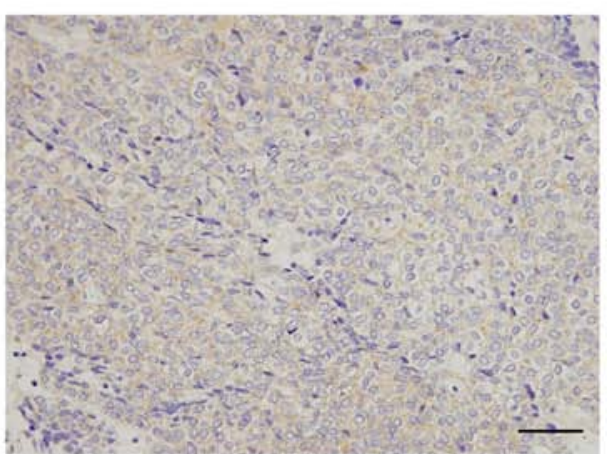

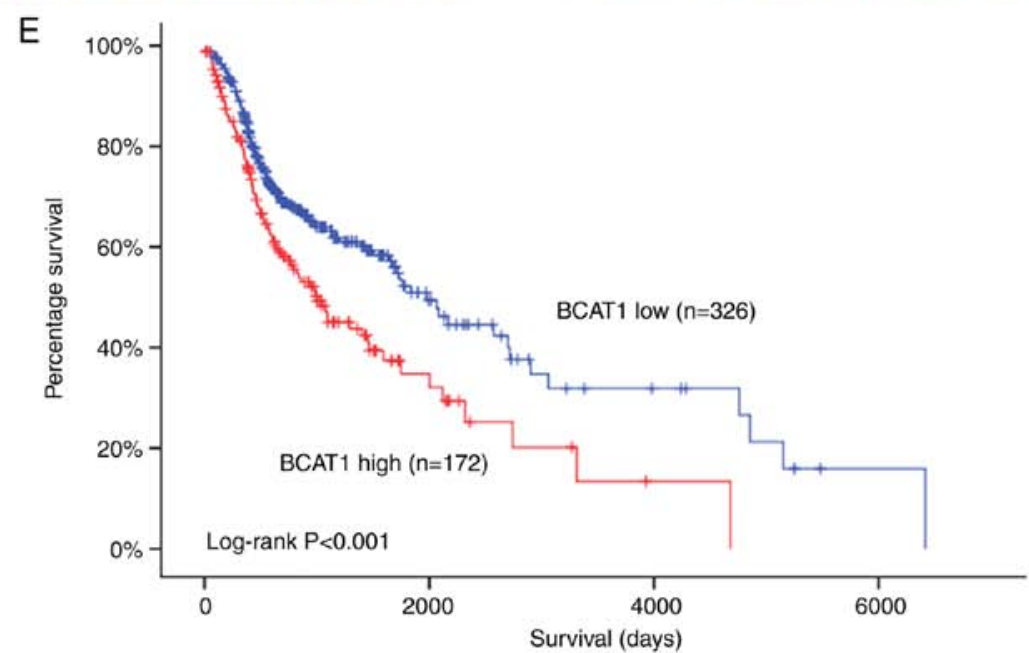

Figure 1. BCAT1 expression is upregulated in HNSCC tissues. (A) BCAT1 weak/negative staining in normal throat tissue. (B) BCAT1 negative staining in normal oral mucosa. (C) BCAT1 strong staining in a case of HNSCC. (D) BCAT1 weak expression in a case of HNSCC. (E) Survival curve of patients with HNSCC exhibiting different levels of BCAT1 expression using the Cancer Genome Atlas data. Scale bars, $50 \mu \mathrm{m}$. BCAT1, branched chain amino acid transaminase 1; HNSCC, head and neck squamous cell carcinoma.

Mann-Whitney U tests were used for the comparison of BCAT1 mRNA levels between cancerous and normal tissues. Kruskal-Wallis test with Dunn's multiple comparison post hoc test was used to evaluate the relationship between clinical $\mathrm{T}$ stage and BCAT1 expression. For comparison of GLUT1 mRNA data among multiple groups, ANOVA analysis with Tukey's multiple comparisons post hoc test was performed. Other data were compared using Student's t-test. $\mathrm{P}<0.05$ was considered to indicate a statistically significant difference.

\section{Results}

BCAT1 is upregulated in HNSCC. BCAT1 protein levels were examined in 106 cases of HNSCC and 23 normal tissues using immunohistochemistry. BCAT1 protein was detected in normal throat tissues (Fig. 1A) and oral mucosa (Fig. 1B). BCAT1 showed negative/low staining in most normal cells. In contrast, BCAT1 protein expression was elevated in tumor tissues (Fig. 1C and D). In 106 cases examined, $52.8 \%(56 / 106)$ exhibited high BCAT1 expression. As presented in Table I, high BCAT1 expression was positively associated with tumor-node-metastasis (TNM) stage, lymph nodal metastasis and higher $\mathrm{T}$ stage. In addition, a cohort of patients with HNSCC frin TCGA showed that high BCAT1 expression was associated with shorter overall survival (Fig. 1E). TCGA data also indicated that BCAT1 mRNA was higher in HNSCC cases with a higher clinical T stage (Fig. 2A). Data from the Oncomine database were also analyzed. The Ginos, Peng, Sengupta, Toruner and Ye head-neck datasets in Oncomine indicated that BCAT1 
A

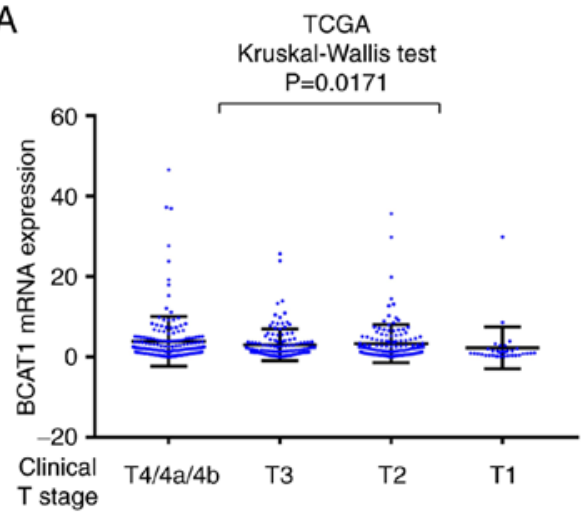

Dunn's multiple comparison test T4 vs T3: $p>0.9999$ T4 vs T2: $p>0.9999$

T4 vs T1: $p=0.0096$ T3 vs T2: $p>0.9999$

T3 vs T1: $p=0.1215$ T2 vs T1: $p=0.0669$

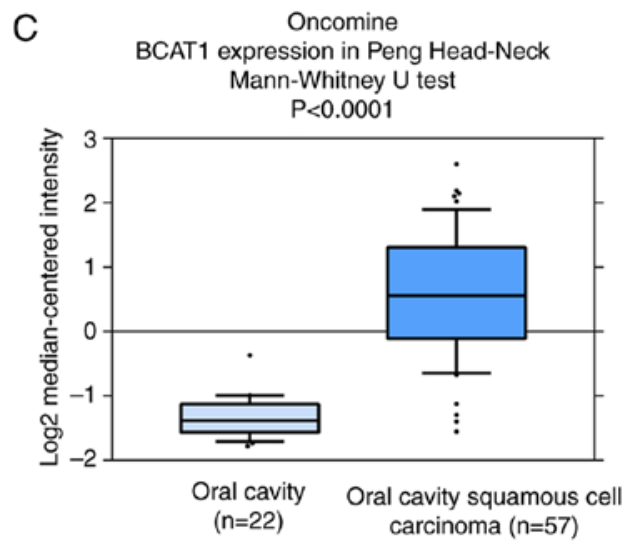

E

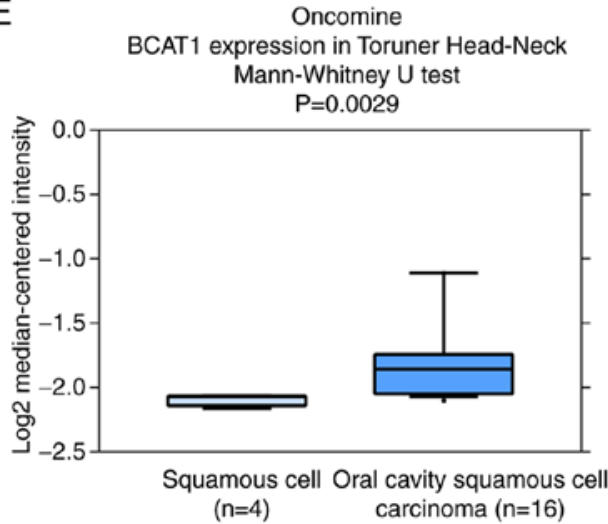

B

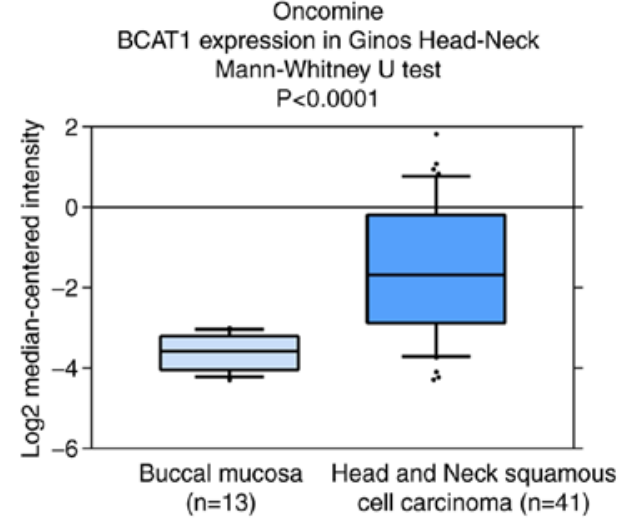

D

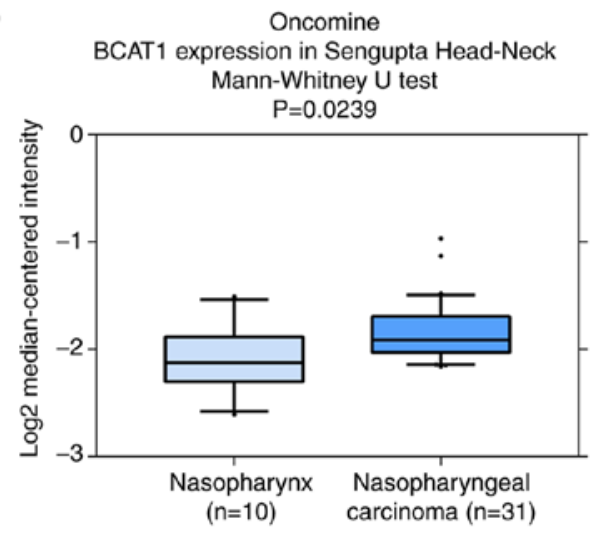

$\mathrm{F}$

Oncomine

BCAT1 expression in Ye Head-Neck Mann-Whitney $U$ test

$P=0.0003$

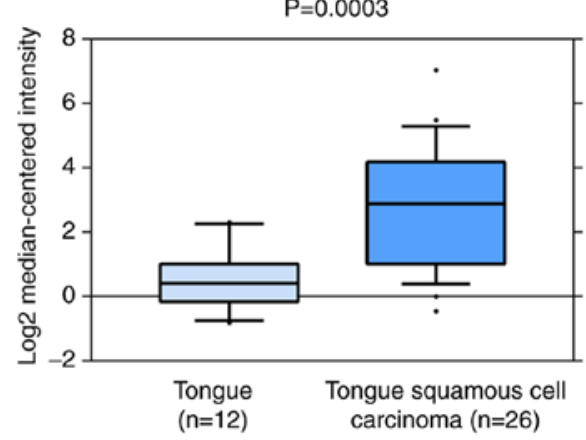

Figure 2. Bioinformatics analysis of BCAT1 expression in HNSCC. (A) Analysis of HNSCC data in TCGA. BCAT1 mRNA expression was higher in HNSCC specimens with higher T stage. Analysis of the (B) Ginos, (C) Peng, (D) Sengupta, (E) Toruner and (F) Ye head-neck datasets in Oncomine, showing that BCAT1 mRNA expression was upregulated in HNSCC tissues compared with normal tissues. BCAT1, branched chain amino acid transaminase 1; HNSCC, head and neck squamous cell carcinoma; TCGA, the Cancer Genome Atlas; T stage, tumor stage.

mRNA was significantly elevated in cancer tissues compared with normal tissues (Fig. 2B-F). These data indicated that BCAT1 expression was upregulated in HNSCC and associated with malignant clinical features.

BCAT1 promotes the proliferation and invasion of FaDu cells. BCAT1 knockdown and overexpression were performed in FaDu cells. The transfection efficiencies of BCAT1 siRNA and overexpression vector were validated via western blotting and RT-qPCR (Fig. 3A). CCK-8 assays demonstrated that BCAT1 knockdown downregulated the proliferation rate of FaDu cells, while BCAT1 overexpression increased the cellular proliferation rate (Fig. 3B). Colony formation assays demonstrated that BCAT1 knockdown decreased colony formation ability, while BCAT1 overexpression upregulated colony formation ability (Fig. 3C).

As there was a significant association between high BCAT1 levels and nodal metastasis in clinical specimens, the invasive ability of FaDu cells was determined after BCAT1 overexpression and knockdown. BCAT1 depletion inhibited 
A

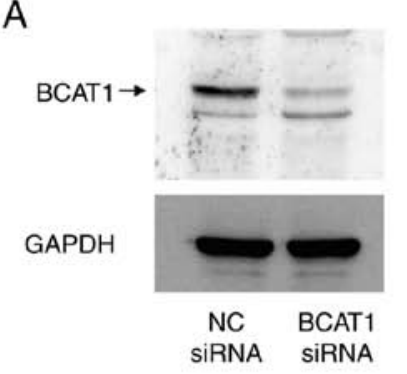

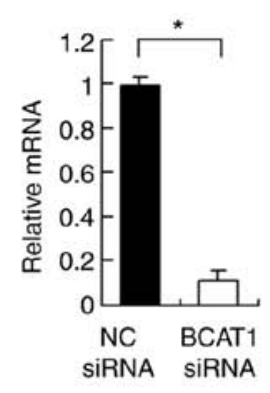

B

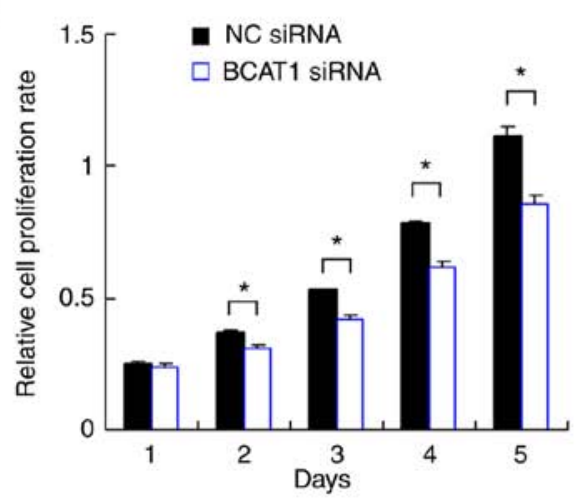

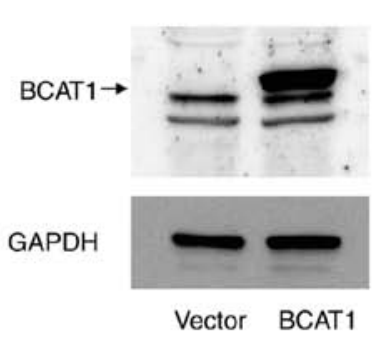
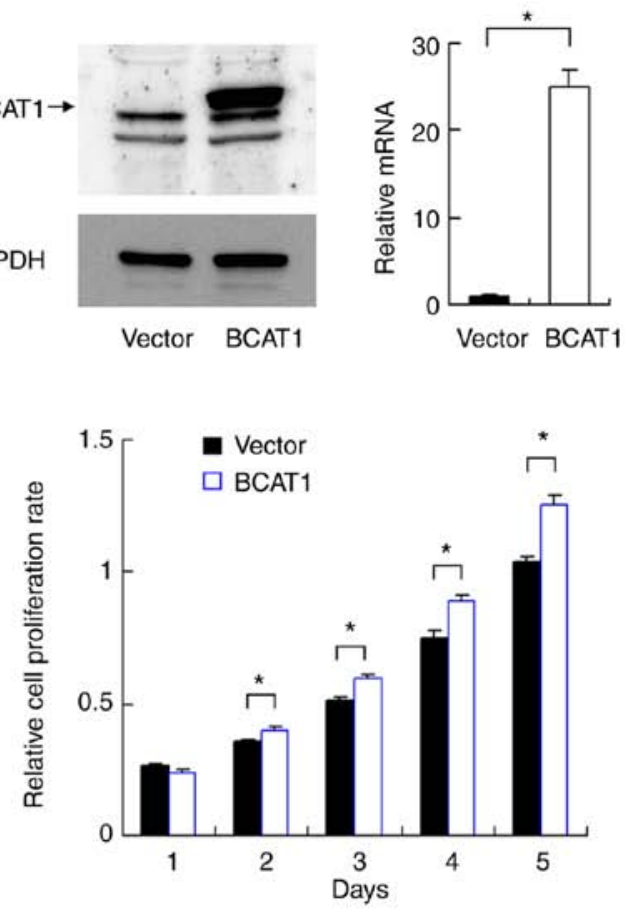

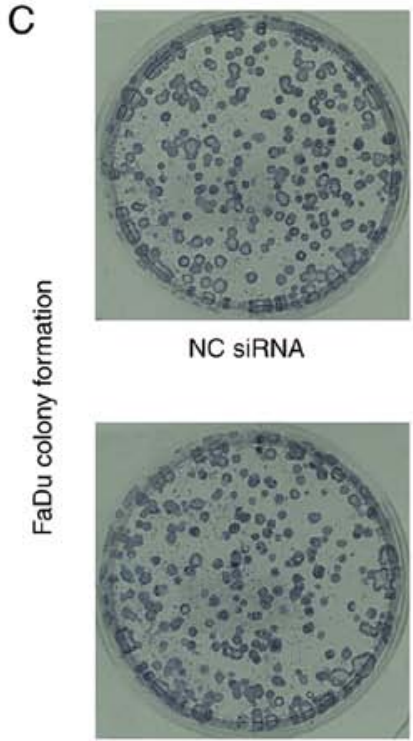

Vector

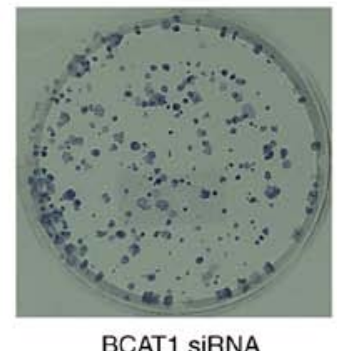

BCAT1 SIRNA

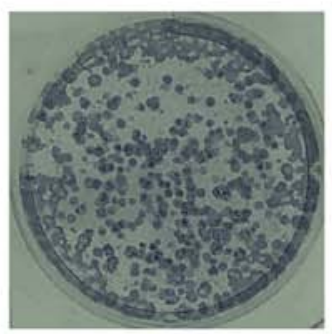

BCAT1
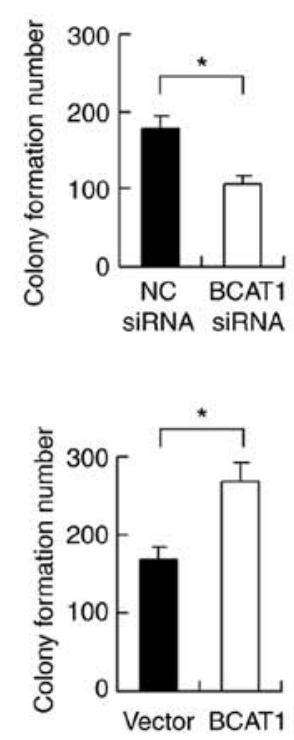

Figure 3. BCAT1 promotes head and neck squamous cell carcinoma cell proliferation. (A) Transfection of pCMV6-BCAT1 plasmid upregulates BCAT1 protein and mRNA levels, whereas BCAT1 siRNA downregulates BCAT1 protein and mRNA levels in FaDu cells. (B) Cell Counting Kit-8 assay analysis of the effects of BCAT1 upregulation and downregulation of FaDu cell proliferation. (C) Colony formation analysis of FaDu cells transfected with BCAT1 plasmid or siRNA. "P<0.05. BCAT1, branched chain amino acid transaminase 1; siRNA, small interfering RNA; NC, negative control.

invasion, while BCAT1 overexpression increased the invasive ability of FaDu cells (Fig. 4A).

BCAT1 regulates chemosensitivity and mitochondrial function. The role of BCAT1 in chemosensitivity was also investigated. Cisplatin $(2 \mu \mathrm{M})$ was used to treat $\mathrm{FaDu}$ cells after BCAT1 siRNA or plasmid transfection. CCK-8 assays showed that BCAT1 knockdown increased cisplatin-mediated inhibition of cells after 24 and $48 \mathrm{~h}$ of treatment, whereas BCAT1 overexpression induced opposing effects (Fig. 4B). Annexin V/PI staining showed that BCAT1 knockdown upregulated the levels of cisplatin-induced apoptosis after
$24 \mathrm{~h}$ of cisplatin treatment. BCAT1 overexpression reduced the apoptosis rate, suggesting that BCAT1 could induce resistance to chemotherapeutic drugs (Fig. 4C).

Chemotherapeutic drugs such as cisplatin can reduce the mitochondrial membrane potential, which plays a pivotal role in cell survival, as its loss induces apoptosis (16). FaDu cells were transfected with pCMV6-BCAT1, BCAT1 siRNA or corresponding negative controls, and were treated with subsequently treated with cisplatin. Then, JC-1 staining was performed to determine the change in $\Delta \psi \mathrm{m}$. JC-1 exhibits red fluorescence in normal conditions but exhibits green fluorescence when $\Delta \psi \mathrm{m}$ is downregulated. BCAT1 siRNA increased 
A

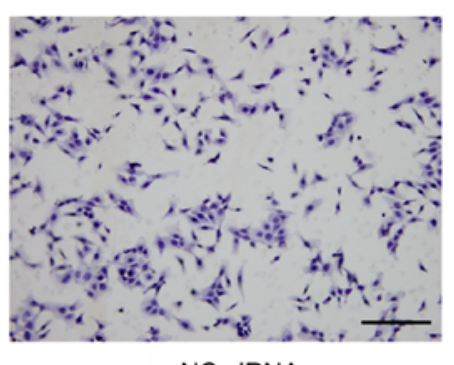

NC SiRNA

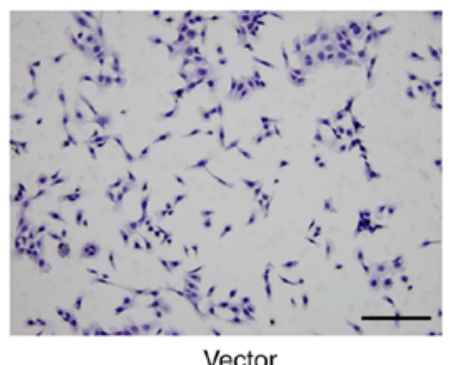

Vector

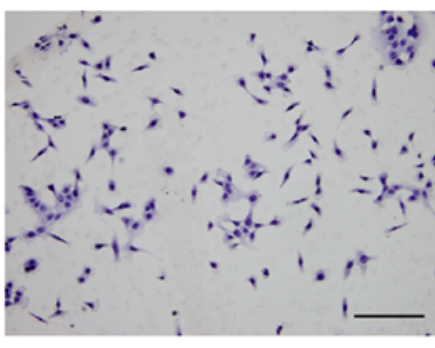

BCAT1 SIRNA

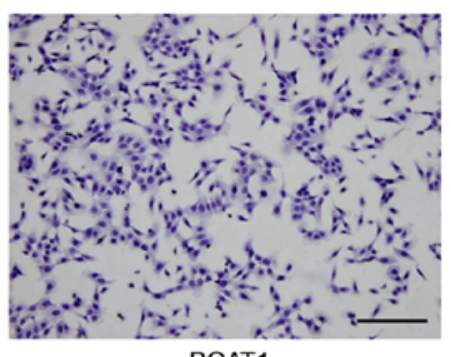

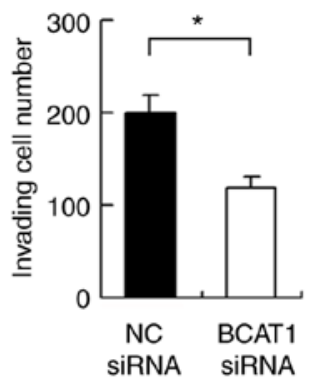

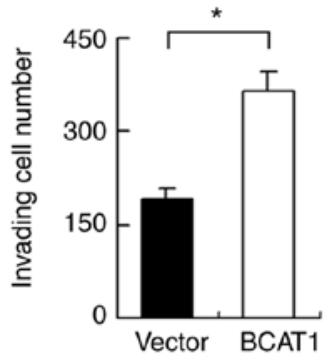

B

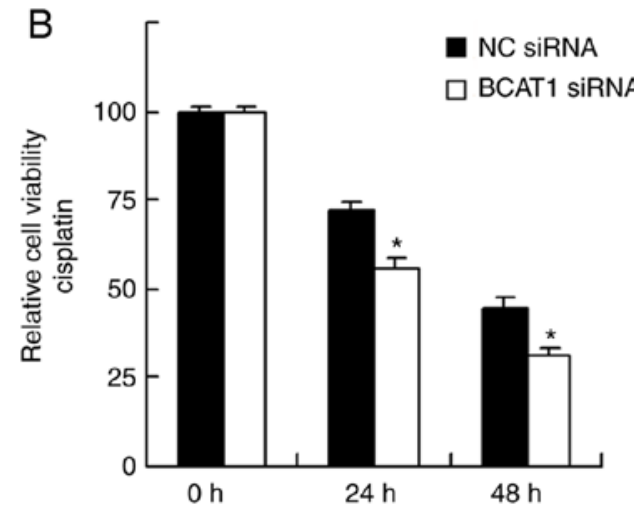

C

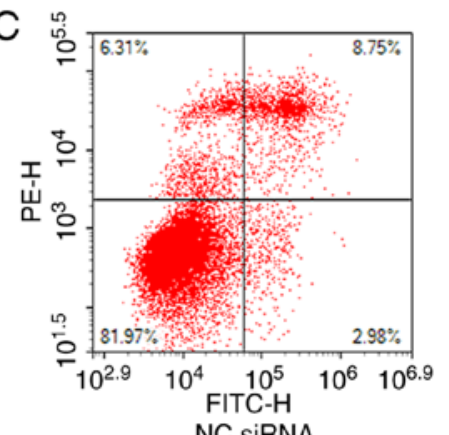

NC SiRNA

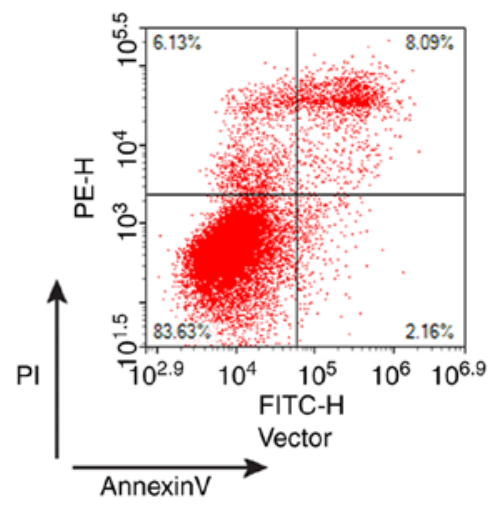

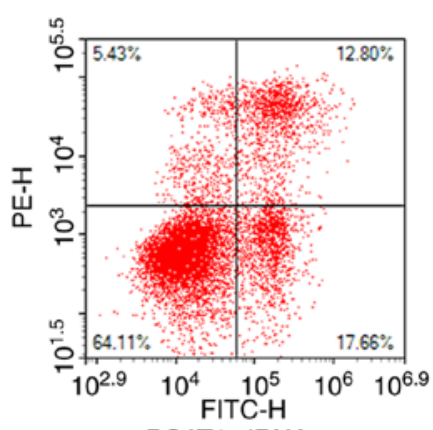

BCAT1 SIRNA

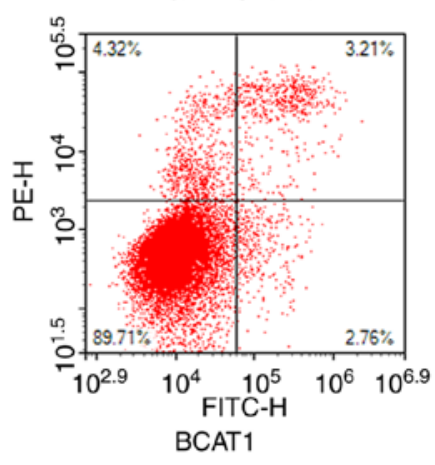

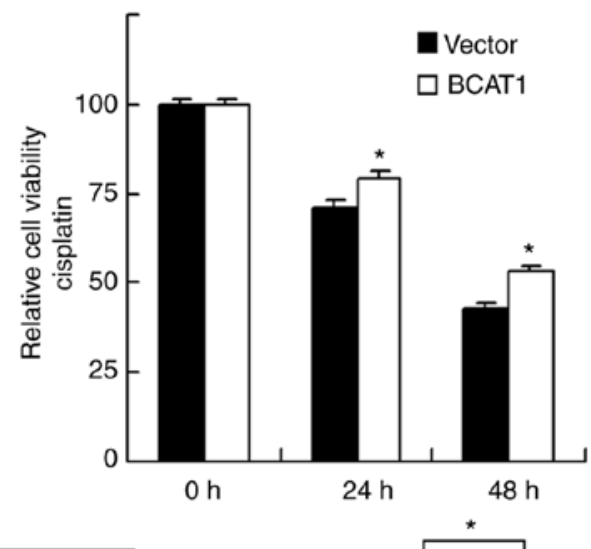
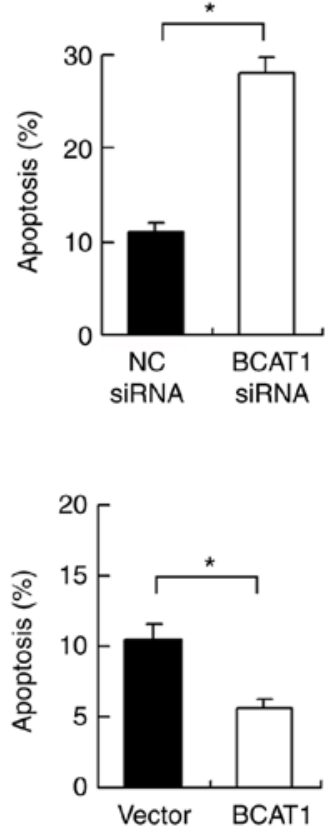

Figure 4. BCAT1 promotes head and neck squamous cell carcinoma cell invasion and cisplatin resistance. (A) BCAT1 overexpression increases the number of invading cells, while BCAT1 knockdown decreases the number of invading cells. Scale bars, $50 \mu$ m. (B) Cell Counting Kit- 8 assays demonstrating that BCAT1 overexpression decreases the efficacy of cisplatin, whereas BCAT1 knockdown increases cisplatin-induced cell inhibition. (C) Annexin V/PI staining showing that BCAT1 overexpression reduces the levels of cisplatin-induced apoptosis. ${ }^{*} \mathrm{P}<0.05$ vs. negative control siRNA or vector. BCAT1, branched chain amino acid transaminase 1; PI, propidium iodide; siRNA, small interfering RNA; NC, negative control. 

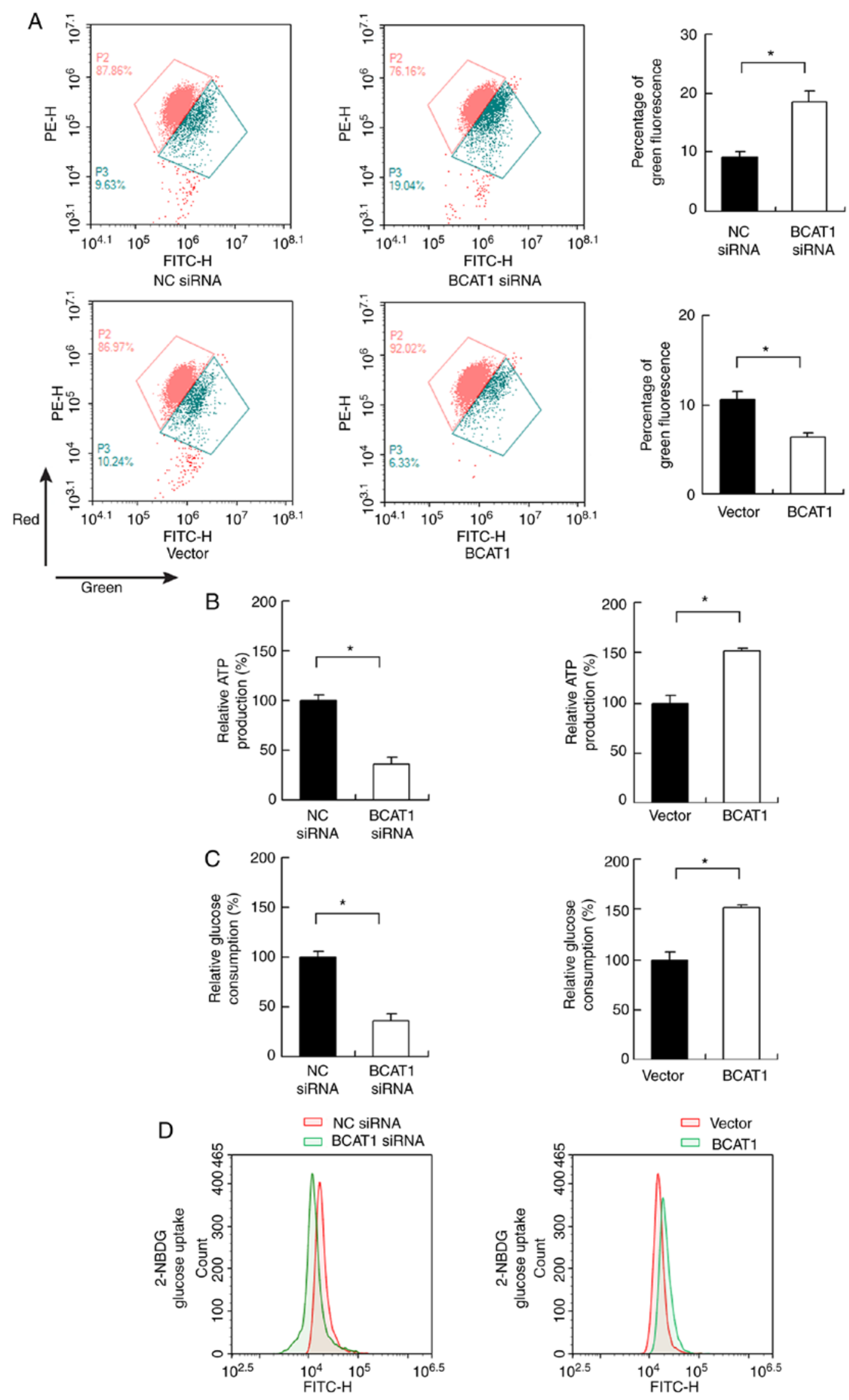

Figure 5. BCAT1 regulates $\Delta \psi \mathrm{m}$, ATP production and glucose uptake in head and neck squamous cell carcinoma cells. (A) JC-1 staining demonstrating that changes in BCAT1 expression alter $\Delta \psi \mathrm{m}$ in FaDu cells following treatment with $2 \mu \mathrm{M}$ cisplatin. (B) BCAT1 overexpression increases relative ATP production, whereas BCAT1 knockdown decreases ATP production in FaDu cells. (C) BCAT1 overexpression increases the relative glucose consumption rate, whereas BCAT1 knockdown decreases the glucose consumption rate of FaDu cells. (D) 2-NBDG glucose uptake in FaDu cells following upregulation or downregulation of BCAT1 expression. ${ }^{*} \mathrm{P}<0.05 . \Delta \psi \mathrm{m}$, mitochondrial membrane potential; BCAT1, branched chain amino acid transaminase 1; siRNA, small interfering RNA; NC, negative control. 
A

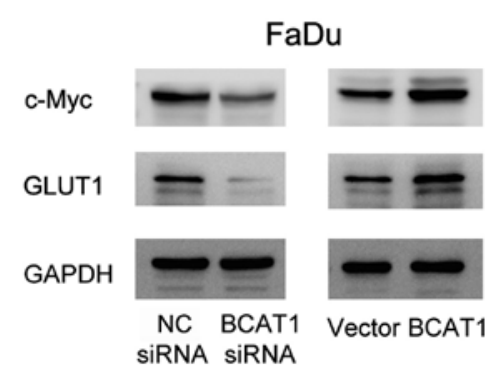

C

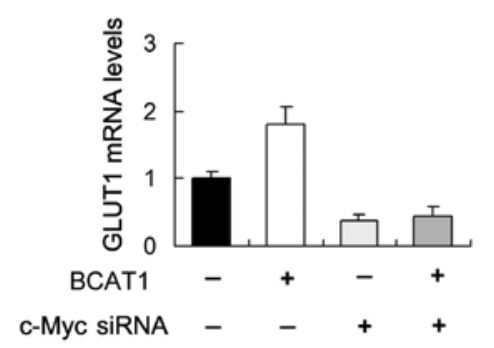

D
B
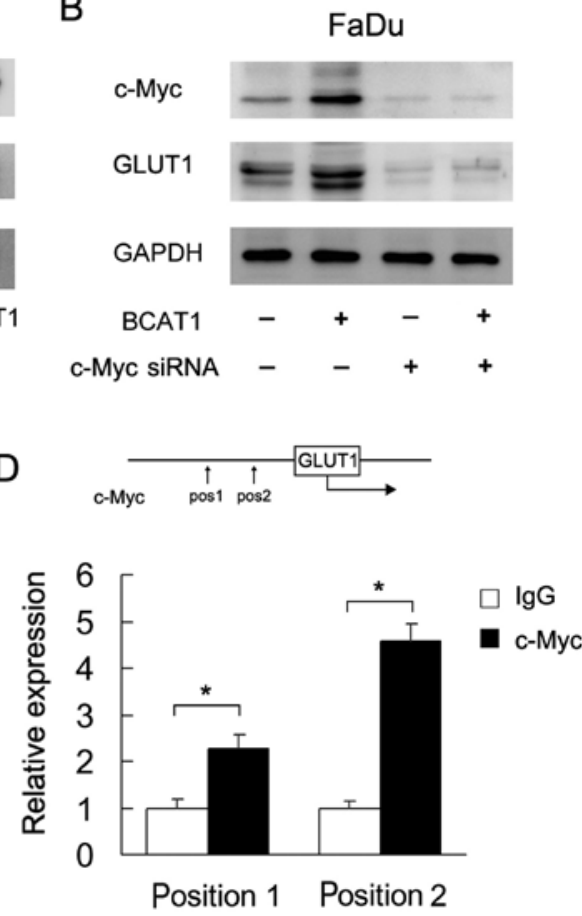

Figure 6. BCAT1 upregulates c-Myc and GLUT1 expression. (A) Western blot analysis of c-Myc and GLUT1 protein expression following transfection with BCAT1 plasmid or siRNA. Effects of BCAT1 overexpression and c-Myc siRNA on the (B) protein and (C) mRNA expression of c-Myc and GLUT1. (D) Chromatin immunoprecipitation analysis of interactions between c-Myc and the GLUT1 promoter. "P<0.05. BCAT1, branched chain amino acid transaminase 1; GLUT1, glucose transporter 1; siRNA, small interfering RNA; NC, negative control.

the percentage of green staining, while BCAT1 overexpression decreased green staining, suggesting that BCAT1 plays an important role in maintaining normal $\Delta \psi \mathrm{m}$ (Fig. 5A).

BCAT1 regulates ATP production and glucose uptake. Cancer cells adopt glucose metabolism to produce energy (ATP), which is essential for cancer cell survival and proliferation. Changes in ATP production were measured in $\mathrm{FaDu}$ cells, and it found that BCAT1 knockdown decreased ATP levels, whereas BCAT1 overexpression upregulated ATP levels (Fig. 5B). Subsequently, changes in glucose consumption and uptake were evaluated. As shown in Fig. 5C, BCAT1 siRNA reduced glucose consumption, whereas BCAT1 overexpression increased it. 2-NBDG uptake showed that BCAT1 knockdown inhibited the levels of glucose uptake, while BCAT1 overexpression upregulated glucose uptake (Fig. 5D).

BCAT1 regulates $c-M y c / G L U T 1$ signaling. To explore the mechanism underlying the role of BCAT1 in HNSCC, several potentially associated proteins (including GLUT1, GLUT2, GLUT3 and GLUT4) were screened. Of these, western blotting showed that BCAT1 positively regulated GLUT1 protein expression (Fig. 6A). It was also found that BCAT1 siRNA downregulated c-Myc, which was upregulated by BCAT1 overexpression. c-Myc has been reported as a transcriptional regulator of glucose metabolism (17). To validate the association between c-Myc and BCAT1-induced GLUT1 upregulation, c-Myc siRNA was used in FaDu cells co-transfected with the BCAT1 plasmid. c-Myc siRNA suppressed the protein and mRNA expression of GLUT1 (Fig. 6B and C).
c-Myc siRNA also attenuated the effect of BCAT1 plasmid on GLUT1 expression at both the protein and mRNA levels (Fig. 6B and C). Furthermore, it was investigated as to whether c-Myc binds to the GLUT1 promoter. Binding sites for c-Myc in the GLUT1 promoter were predicted using TRANSFAC. Binding of c-Myc to GLUT1 promoter was examined using ChIP assays followed by RT-qPCR (Fig. 6D). The results indicated that the effect of BCAT1 on GLUT1 was at least partly dependent on c-Myc.

\section{Discussion}

In the present study, it was demonstrated that BCAT1 was upregulated in human HNSCC and promoted cell proliferation, invasion, cisplatin resistance and glucose uptake, potentially via c-Myc/GLUT1 signaling. Previous studies have focused on the role of BCAT1 in BCAA metabolism $(11,18)$, but its potential roles in other pathways have not been fully explored. The present data highlighted the role of BCAT1 in c-Myc/GLUT1 signaling and HNSCC progression.

Recent studies have shown that BCAT1 acts as a cancer-related protein $(9-12,19)$. A recent study showed that BCAT1 was overexpressed in human non-small cell lung cancer (NSCLC) tissues and cell lines (20). To date, there has been no such study regarding BCAT1 in human HNSCC, to the best of the authors' knowledge. The present findings showed that BCAT1 upregulation was associated with TNM stage and nodal status, which was supported by analyses of data from TCGA and Oncomine. TCGA data also showed that BCAT1 levels were associated with poor prognosis, suggesting the potential use of BCAT1 as a clinical indicator 
of malignant HNSCC. It was noted that BCAT1 overexpression was not associated with the differentiation of HNSCC. Generally, poorly differentiated HNSCC is biologically more aggressive and tends to metastasize to regional lymph nodes (21). However, HNSCC is a heterogeneous cancer with a variety of histological subtypes. Differentiation is not generally considered to be a direct indicator of malignancy, and nodal/distal metastasis and clinical stage are more useful for predicting patient prognosis $(22,23)$.

CCK-8 and colony formation assays demonstrated that BCAT1 overexpression promoted in vitro cell proliferation. Matrigel invasion assays showed that BCAT1 facilitated HNSCC cell invasion. In addition, it was determined that BCAT1 overexpression decreased cisplatin sensitivity and reduced cisplatin-induced apoptosis. Mitochondrial function serves an important role in regulating chemosensitivity (24). A decrease in $\Delta \psi \mathrm{m}$ can induce mitochondrial permeability, which accelerates cytochrome $c$ release and triggers apoptosis (25-29). It was revealed that BCAT1 overexpression preserved $\Delta \psi \mathrm{m}$ after cisplatin treatment, suggesting its role as a protector of mitochondrial function.

Mitochondrial balance in tumor cells relies on energy supply $(30,31)$. Glucose uptake is an important step during ATP production. It was demonstrated that BCAT1 overexpression increased glucose uptake and consumption, and upregulated ATP production, revealing BCAT1 as a positive regulator of glucose metabolism. It should be noted that while BCAA metabolism may play a part in cancer growth, other signaling pathways may also contribute to malignant features induced by BCAT1. These data therefore provide evidence for novel roles of BCAT1 in regulating glucose metabolism in HNSCC cells.

In accordance with the increased glucose uptake, it was further demonstrated that BCAT1 upregulated GLUT1 expression. GLUT family proteins, which mediate the transport of glucose across membranes, were reported to be elevated in human cancers $(28,32)$. It has been reported that GLUT1 regulated the proliferation and survival of HNSCC cells (33). It was also found that BCAT1 upregulated c-Myc expression. c-Myc has been reported to control genes regulating glucose metabolism (34). Using siRNA-mediated knockdown of c-Myc, it was shown that c-Myc mediated BCAT1-induced GLUT1 upregulation, which was further supported by ChIP data showing that c-Myc directly bound to the GLUT1 promoter in FaDu cells. A recent study in NSCLC also indicated the regulatory role of BCAT1 on c-Myc, potentially via regulation of Wnt signaling (20). BCAT1 has also been reported as a target gene of c-Myc (35). Thus, there may be a positive feedback loop between BCAT1 and c-Myc in HNSCC. Collectively, the present findings revealed a link between BCAT1, c-Myc and GLUT1.

In conclusion, the present study revealed biological roles of BCAT1 in human HNSCC. The results showed that BCAT1 overexpression promoted proliferation, invasion, cisplatin resistance and glucose uptake in HNSCC cells. Its oncogenic effects may be associated with its interactions with c-Myc/GLUT1 signaling, suggesting the therapeutic possibility of targeting BCAT1 in HNSCC.

\section{Acknowledgements}

Not applicable.

\section{Funding}

The present study was funded by The Natural Science Foundation of Liaoning (grant no. 20180540097).

\section{Availability of data and materials}

The datasets used and/or analyzed during the current study are available from the corresponding author upon reasonable request.

\section{Authors' contributions}

HW and FW performed the experiments and drafted the manuscript. HW and WO collected and analyzed the data. XJ and YW conceived, designed and supervised the study, and drafted the manuscript. All authors read and approved the final manuscript and agree to be responsible for all aspects of the work in ensuring that the accuracy or integrity of any part of the work are appropriately investigated and resolved.

\section{Ethics approval and consent to participate}

The present study was approved by the Institute Research Ethics Committee of the First Affiliated Hospital of China Medical University and written informed consent was obtained from all patients involved.

\section{Patient consent for publication}

Not applicable.

\section{Competing interests}

The authors declare that they have no competing interests.

\section{References}

1. Chen W, Zheng R, Baade PD, Zhang S, Zeng H, Bray F, Jemal A, Yu XQ and He J: Cancer statistics in China, 2015. CA Cancer J Clin 66: 115-132, 2016.

2. Siegel RL, Miller KD and Jemal A: Cancer statistics, 2015. CA Cancer J Clin 65: 5-29, 2015.

3. Leemans CR, Braakhuis BJ and Brakenhoff RH: The molecular biology of head and neck cancer. Nat Rev Cancer 11: 9-22, 2011.

4. Yang CX, Sedhom W, Song J and Lu SL: The role of MicroRNAs in recurrence and metastasis of head and neck squamous cell carcinoma. Cancers (Basel) 11: 395, 2019.

5. Eden A and Benvenisty N: Involvement of branched-chain amino acid aminotransferase (Bcat1/Eca39) in apoptosis. FEBS Lett 457: 255-261, 1999

6. Ben-Yosef T, Eden A and Benvenisty N: Characterization of murine BCAT genes: Bcat1, a c-Myc target, and its homolog, Bcat2. Mamm Genome 9: 595-597, 1998.

7. Garcia-Espinosa MA, Wallin R, Hutson SM and Sweatt AJ: Widespread neuronal expression of branched-chain aminotransferase in the CNS: Implications for leucine/glutamate metabolism and for signaling by amino acids. J Neurochem 100: 1458-1468, 2007.

8. Tonjes M, Barbus S, Park YJ, Wang W, Schlotter M, Lindroth AM, Pleier SV, Bai AHC, Karra D, Piro RM, et al: BCAT1 promotes cell proliferation through amino acid catabolism in gliomas carrying wild-type IDH1. Nat Med 19: 901-908, 2013.

9. Zhang L and Han J: Branched-chain amino acid transaminase 1 (BCAT1) promotes the growth of breast cancer cells through improving mTOR-mediated mitochondrial biogenesis and function. Biochem Biophys Res Commun 486: 224-231, 2017. 
10. Raffel S, Falcone M, Kneisel N, Hansson J, Wang W, Lutz C, Bullinger L, Poschet G, Nonnenmacher Y, Barnert A, et al: BCAT1 restricts $\alpha \mathrm{KG}$ levels in AML stem cells leading to IDHmut-like DNA hypermethylation. Nature 551: 384-388, 2017.

11. Hattori A, Tsunoda M, Konuma T, Kobayashi M, Nagy T, Glushka J, Tayyari F, McSkimming D, Kannan N, Tojo A, et al: Cancer progression by reprogrammed BCAA metabolism in myeloid leukaemia. Nature 545: 500-504, 2017.

12. Qi LN, Xiang BD, Wu FX, Ye JZ, Zhong JH, Wang YY, Chen YY, Chen ZS, Ma L, Chen J, et al: Circulating tumor cells undergoing EMT provide a metric for diagnosis and prognosis of patients with hepatocellular carcinoma. Cancer Res 78: 4731-4744, 2018.

13. Wang Z, Jensen MA and Zenklusen JC: A practical guide to the cancer genome atlas (TCGA). Methods Mol Biol 1418: 111-141, 2016.

14. Rhodes DR, Kalyana-Sundaram S, Mahavisno V, Varambally R, Yu J, Briggs BB, Barrette TR, Anstet MJ, Kincead-Beal C, Kulkarni P, et al: Oncomine 3.0: Genes, pathways, and networks in a collection of 18,000 cancer gene expression profiles. Neoplasia 9: 166-180, 2007.

15. Livak KJ and Schmittgen TD: Analysis of relative gene expression data using real-time quantitative PCR and the 2(-Delta Delta C(T)) method. Methods 25: 402-408, 2001

16. Erxleben A: Mitochondria-targeting anticancer metal complexes. Curr Med Chem 26: 694-728, 2019.

17. Marbaniang C and Kma L: Dysregulation of glucose metabolism by oncogenes and tumor suppressors in cancer cells. Asian Pac J Cancer Prev 19: 2377-2390, 2018.

18. Gu Z, Liu Y, Cai F, Patrick M, Zmajkovic J, Cao H, Zhang Y, Tasdogan A, Chen M, Qi L, et al: Loss of EZH2 reprograms BCAA metabolism to drive leukemic transformation. Cancer Discov 9: 1228-1247, 2019.

19. Xu Y, Yu W, Yang T, Zhang M, Liang C, Cai X and Shao Q: Overexpression of BCAT1 is a prognostic marker in gastric cancer. Hum Pathol 75: 41-46, 2018.

20. Lin X, Tan S, Fu L and Dong Q: BCAT1 overexpression promotes proliferation, invasion, and wnt signaling in non-small cell lung cancers. Onco Targets Therapy 13: 3583-3594, 2020.

21. Fortin A, Couture C, Doucet R, Albert M, Allard J and Tetu B: Does histologic grade have a role in the management of head and neck cancers? J Clin Oncol 19: 4107-4116, 2001.

22. Economopoulou P, de Bree R, Kotsantis I and Psyrri A: Diagnostic tumor markers in head and neck squamous cell carcinoma (HNSCC) in the clinical setting. Front Oncol 9: 827, 2019.

23. Bossi P, Alfieri S, Strojan P, Takes RP, López F, Mäkitie A Saba NF, Rodrigo JP, Bradford C, Suarez C, et al: Prognostic and predictive factors in recurrent and/or metastatic head and neck squamous cell carcinoma: A review of the literature. Crit Rev Oncol Hematol 137: 84-91, 2019.
24. Fu L, Dong Q, He J, Wang X, Xing J, Wang E, Qiu X and Li Q: SIRT4 inhibits malignancy progression of NSCLCs, through mitochondrial dynamics mediated by the ERK-Drp1 pathway. Oncogene 36: 2724-2736, 2017.

25. Guerra F, Arbini AA and Moro L: Mitochondria and cancer chemoresistance. Biochim Biophys Acta Bioenerg 1858: 686-699, 2017.

26. Kim JS, Lee JM, Chwae YJ, Kim YH, Lee JH, Kim K, Lee TH, Kim SJ and Park JH: Cisplatin-induced apoptosis in Hep3B cells: Mitochondria-dependent and -independent pathways. Biochem Pharmacol 67: 1459-1468, 2004.

27. Zhao W, You CC, Zhuang JP, Zu JN, Chi ZY, Xu GP and Yan JL: Viability inhibition effect of gambogic acid combined with cisplatin on osteosarcoma cells via mitochondria-independent apoptotic pathway. Mol Cell Biochem 382: 243-252, 2013.

28. Li H, Fu L, Liu B, Lin X, Dong Q and Wang E: Ajuba overexpression regulates mitochondrial potential and glucose uptake through YAP/Bcl-xL/GLUT1 in human gastric cancer. Gene 693: $16-24,2019$.

29. Battogtokh G, Cho YY, Lee JY, Lee HS and Kang HC: Mitochondrial-Targeting anticancer agent conjugates and nanocarrier systems for cancer treatment. Front Pharmacol 9: 922, 2018.

30. Horbay R and Bilyy R: Mitochondrial dynamics during cell cycling. Apoptosis 21: 1327-1335, 2016.

31. Stockburger C, Miano D, Pallas T, Friedland K and Muller WE: Enhanced neuroplasticity by the metabolic enhancer piracetam associated with improved mitochondrial dynamics and altered permeability transition pore function. Neural Plast 2016: 8075903, 2016.

32. Zambrano A, Molt M, Uribe E and Salas M: Glut 1 in cancer cells and the inhibitory action of resveratrol as a potential therapeutic strategy. Int J Mol Sci 20: 3374, 2019.

33. Li S, Yang X, Wang P and Ran X: The effects of GLUT1 on the survival of head and neck squamous cell carcinoma. Cell Physiol Biochem 32: 624-634, 2013.

34. Osthus RC, Shim H, Kim S, Li Q, Reddy R, Mukherjee M, Xu Y, Wonsey D, Lee LA and Dang CV: Deregulation of glucose transporter 1 and glycolytic gene expression by c-Myc. J Biol Chem 275: 21797-21800, 2000.

35. Zhou W, Feng X, Ren C, Jiang X, Liu W, Huang W, Liu Z, Li Z, Zeng L, Wang L, et al: Over-expression of BCAT1, a c-Myc target gene, induces cell proliferation, migration and invasion in nasopharyngeal carcinoma. Mol Cancer 12: 53, 2013.

(i) This work is licensed under a Creative Commons Attribution-NonCommercial-NoDerivatives 4.0 International (CC BY-NC-ND 4.0) License. 